\title{
16
}

\section{The User`s Guide to the Light-sheet Galaxy}

Alessandro Ciccarelli, Alexandra Palmer, Camille Charoy, Dan Gunton, David Barry, Deborah Aubyn, Donald Bell, Fabrice Prin, Matt Rensahw, Rocco D`Antuono, Todd Fallesen, Kurt Anderson The Francis Crick Institute, United Kingdom

\section{Abstract Text}

Light-sheet microscopy is a convenient imaging approach for studying a wide range of biological samples, from single cells layers to whole adult organisms. This technology is relatively young and still not very widely available in light microscopy facilities, yet it is proving to be a valuable asset for a continuously growing range of biological samples. The "light-sheet journey" (from the sample to the data) consists of multiple steps (sample preparation, sample mounting, imaging and data processing) that can be long and different for each set of biological questions. Finding the best settings for imaging a new sample can be a lengthy process, involving manufacturing of sample holders, testing different illumination/detection settings, and optimizing sample preparation protocols (e.g., tissue clearing) and object segmentation. We believe that having a detailed and easily accessible resource about how to image specific samples with light-sheet would help scientists who want to start using this technology. At the Francis Crick Institute, a large research biomedical centre hosting more than 120 research groups, we have currently installed two light-sheet microscopes: the Bruker-Luxendo MuVi SPIM and the Miltenyi-LaVision Biotec UMII. After 3 years of light-sheet imaging, we have acquired a good expertise about successfully imaging a wide range of samples (e.g. zebrafish/mouse live and fixed embryos, mouse bones/other organs, Drosophila larvae, ...) and we are starting to write some protocols to help new users in imaging with this technology. The database that we are producing contains sample-specific mounting/imaging protocols, a description of new sample holders that we have designed, general tools to make easier the sample mounting (PDMS moulds, concave metal mounting dishes, ...), and ad-on modules for 
the microscopes (e.g. web-cam for sample loading, ...). In this study we also discuss about pros and cons of the two commercial lightsheet microscopes that we are currently using. These resources will be continuously updated and should a be useful resource for Crick scientists, the light-sheet community and light microscopy facility staff. 